
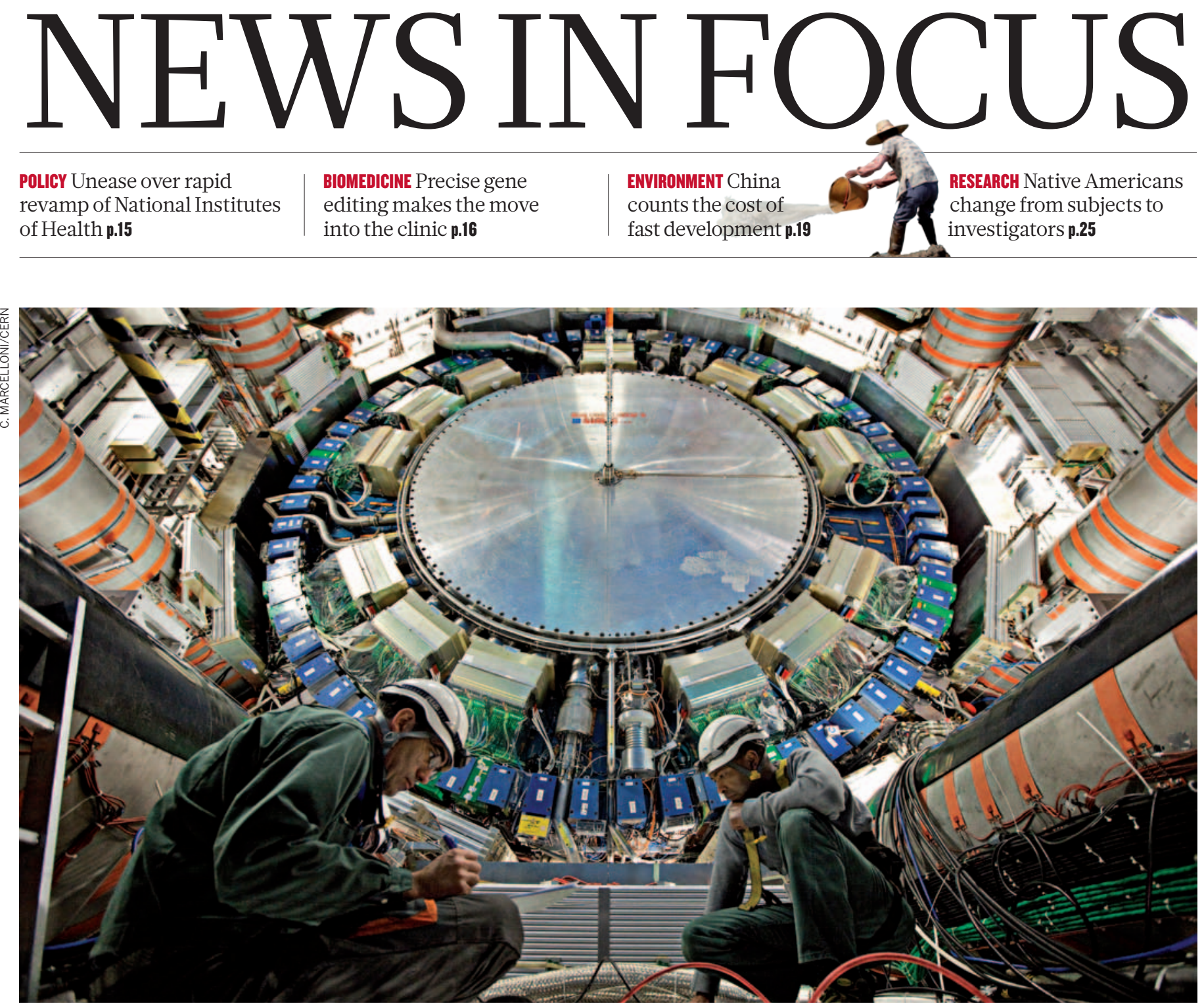

“Any squarks in here?" The ATLAS detector (above) at the Large Hadron Collider has failed to find predicted 'super partners' of fundamental particles.

\title{
PHYSICS
}

\section{Beautiful theory collides with smashing particle data}

Latest results from the LHC are casting doubt on the theory of supersymmetry.

BY GEOFF BRUMFIEL

Cऽ】 $\begin{aligned} & \text { onderful, beautiful and unique" } \\ & \text { is how Gordon Kane describes } \\ & \text { supersymmetry theory. Kane, }\end{aligned}$ a theoretical physicist at the University of Michigan in Ann Arbor, has spent about 30 years working on supersymmetry, a theory that he and many others believe solves a host of problems with our understanding of the subatomic world.
Yet there is growing anxiety that the theory, however elegant it might be, is wrong. Data from the Large Hadron Collider (LHC), a 27-kilometre proton smasher that straddles the French-Swiss border near Geneva, Switzerland, have shown no sign of the 'super particles' that the theory predicts ${ }^{1-3}$. "We're painting supersymmetry into a corner," says Chris Lester, a particle physicist $\rightarrow$ NATURE.COM

Read more at

Nature's LHC

special:

www.nature.com/lhc at the University of Cambridge, UK, who works with the LHC's ATLAS detector. Along with the LHC's Compact Muon Solenoid experiment, ATLAS has spent the past year hunting for super particles, and is now set to gather more data when the LHC begins a high-power run in the next few weeks. If the detectors fail to find any super particles by the end of the year, the theory could be in serious trouble.

Supersymmetry (known as SUSY and pronounced 'Susie') emerged in the 1970s as 
- a way to solve a major shortcoming of the standard model of particle physics, which describes the behaviour of the fundamental particles that make up normal matter (see 'The bestiary'). Researchers have now found every particle predicted by the model, save one: the Higgs boson, theorized to help endow other particles with mass.

The Higgs is crucial to the theory, but its predicted mass is subject to wild fluctuations caused by quantum effects from other fundamental particles. Those fluctuations can increase the Higgs' expected mass to a point at which other fundamental particles should be much more massive than they actually are, effectively breaking the standard model. Theorists can eliminate the fluctuations from their equations, but only by setting the Higgs mass to a very precise value - a fraction heavier or lighter and the whole theoretical edifice collapses. Many physicists are uncomfortable with any theory that requires such delicate fine-tuning to work.

SUSY offers an alternative to this 'finetuning' problem. The theory postulates that each regular particle has a heavier supersymmetrical partner, many of which are unstable and rarely interact with normal matter. The quantum fluctuations of the supersymmetrical particles perfectly cancel out those of the regular particles, returning the Higgs boson to an acceptable mass range.

Theorists have also discovered that SUSY can solve other problems. Some of the lightest supersymmetrical particles could be the elusive dark matter that cosmologists have been hunting for since the 1930s. Although it has never been seen, dark matter makes up about $83 \%$ of the matter in the Universe, according to observations of how galaxies move. SUSY can also be used to bring together all the forces except gravity into a single force at high energies, a big step towards a 'theory of everything' that unifies and explains all known physics - one of the ultimate goals of science. Perhaps most important for some theorists, "SUSY is very beautiful mathematically”, says Ben Allanach, a theorist at the University of Cambridge.

SUSY's utility and mathematical grace have instilled a "religious devotion" among its followers, says Adam Falkowski, a theorist at the University of Paris-South in France. But colliders have failed to turn up direct evidence of the super particles predicted by the theory.

\section{THE BESTIARY}

Could shadowy super particles be lurking behind the standard model's observed fundamental particles and forces?
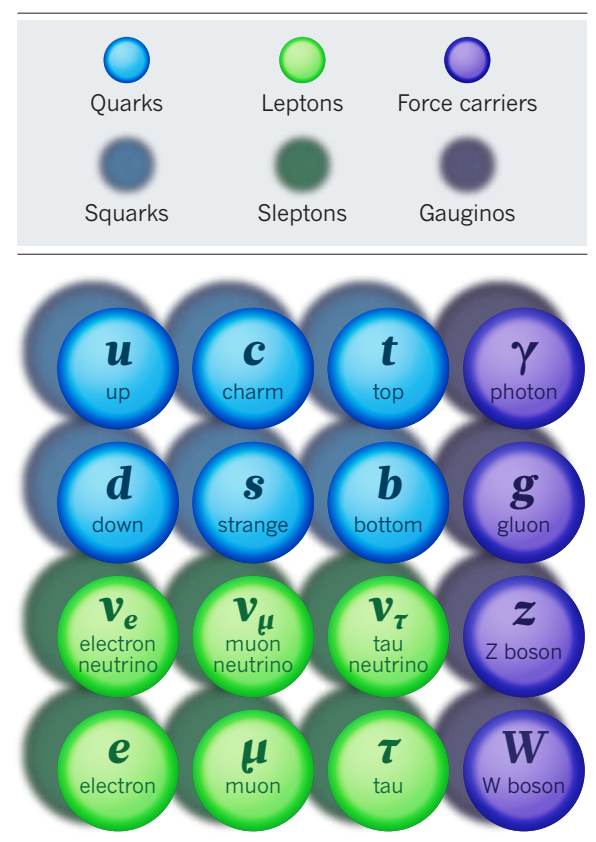

SUSY'S MID-LIFE CRISIS

\begin{tabular}{|c|c|}
\hline $1970-74$ & $\begin{array}{l}\text { Several theorists independently } \\
\text { develop SUSY }\end{array}$ \\
\hline 1981 & $\begin{array}{l}\text { Supersymmetric version of the } \\
\text { standard model proposed }\end{array}$ \\
\hline 1983 & SUSY used to explain dark matter \\
\hline 1990 & $\begin{array}{l}\text { SUSY suggested as a way to unify } \\
\text { electroweak and strong forces }\end{array}$ \\
\hline 2000 & $\begin{array}{l}\text { Large Electron Positron collider (the LHC's } \\
\text { predecessor) fails to find evidence of SUSY } \\
\text { particles called sleptons }\end{array}$ \\
\hline 2008 & $\begin{array}{l}\text { Tevatron sets mass limits on } \\
\text { supersymmetric quarks (squarks) }\end{array}$ \\
\hline 2011 & LHC tightens limits on SUSY masses \\
\hline
\end{tabular}

The Tevatron at the Fermi National Accelerator Laboratory in Batavia, Illinois, for example, has found no evidence of supersymmetrical quarks ('squarks') at masses of up to 379 gigaelectronvolts (energy and mass are used interchangeably in the world of particle physics).

The LHC is now rapidly accumulating data at higher energies, ruling out heavier territory for the super particles. This creates a serious problem for SUSY (see 'SUSY's mid-life crisis'). As the super particles increase in mass, they no longer perfectly cancel out the troubling quantum fluctuations that they were meant to ${ }_{0}$ correct. Theorists can still make SUSY work, but only by assuming very specific masses for the super particles - the kind of fine-tuning exercise that the theory was invented to avoid. As the LHC collects more data, SUSY will require increasingly intrusive tweaks to the masses of the particles.

So far the LHC has doubled the mass limit set by the Tevatron, showing no evidence of squarks at energies up to about 700 gigaelectronvolts. By the end of the year, it will reach 1,000 gigaelectronvolts - potentially ruling out some of the most favoured variations of supersymmetry theory.

"I'm wouldn't say I'm concerned," says John Ellis, a theorist at CERN, Europe's particlephysics lab near Geneva, who has worked on supersymmetry for decades. He says that he will wait until the end of 2012 - once more runs at high energy have been completed - before abandoning SUSY. Falkowski, a long-time critic of the theory, thinks that the lack of detections already suggest that SUSY is dead.

"Privately, a lot of people think that the situation is not good for SUSY," says Alessandro Strumia, a theorist at the University of Pisa in Italy, who recently produced a paper about the impact of the LHC's latest results on the finetuning problem ${ }^{4}$. "This is a big political issue in our field," he adds. "For some great physicists, it is the difference between getting a Nobel prize and admitting they spent their lives on the wrong track." Ellis agrees: "I’ve been working on it for almost 30 years now, and I can imagine that some people might get a little bit nervous."

"Plenty of things will change if we fail to discover SUSY," says Lester. Theoretical physicists will have to go back to the drawing board and find an alternative way to solve the problems with the standard model. That's not necessarily a bad thing, he adds: "For particle physics as a whole it will be really exciting." - SEE EDITORIAL P.6

\footnotetext{
1. ATLAS Collaboration. Preprint at http://arxiv.org/ abs/1102.2357 (2011).

2. CMS Collaboration. Preprint at http://arxiv.org/ abs/1101.1628 (2011).

3. ATLAS Collaboration. Preprint at http://arxiv.org/ abs/1102.5290 (2011).

4. Strumia, A. Preprint at http://arxiv.org/ abs/1101.2195 (2011)
}

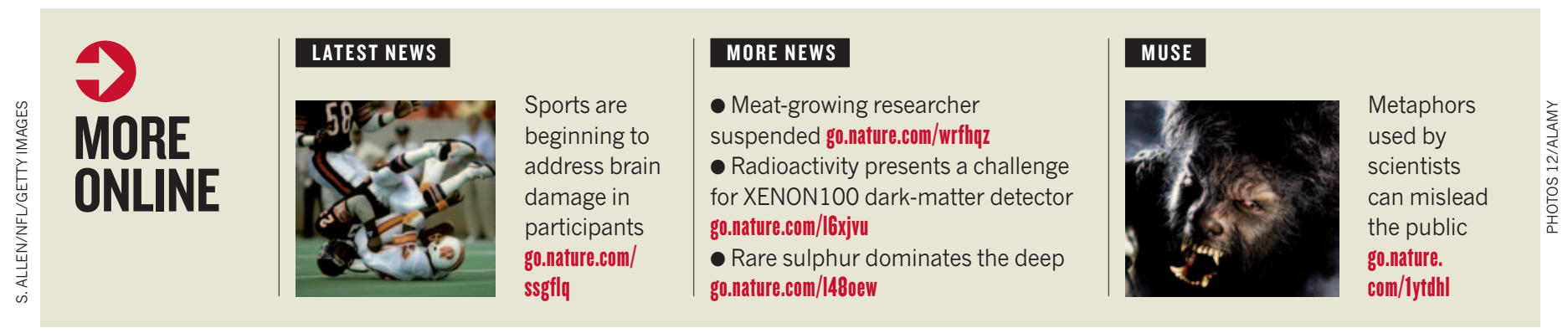

